\title{
Being overweight has limited effect on SCARF osteotomy outcome for hallux valgus correction
}

\author{
Marcin A. Milczarek ${ }^{1}$ - Julia J. Milczarek ${ }^{2}$ - Bartlomiej Tomasik ${ }^{3}$. \\ Przemyslaw Laganowski ${ }^{1} \cdot$ Krzysztof Nowak $^{1} \cdot$ Marcin Domżalski $^{1}$
}

Received: 3 August 2016 / Accepted: 26 January 2017 / Published online: 16 February 2017

(C) The Author(s) 2017. This article is published with open access at Springerlink.com

\begin{abstract}
Purpose The purpose of this study was to investigate the association between body mass index (BMI) and the results of SCARF osteotomy of the first metatarsal for hallux valgus (HV) correction, as the literature on this is scant.

Methods This prospective study was carried out between 2011 and 2015. One hundred and thirty-three patients diagnosed with moderate to severe HV underwent a SCARF corrective osteotomy. We divided the patients into two groups according to their BMI: normal and overweight. Postoperative follow-up was two years. All patients were examined twice by two medical doctors simultaneously: pre-operatively and postoperatively at two years' follow-up. Data collected included biometrical records, X-rays [HV angle (HVA), intermetatarsal angle (IMA), American Orthopaedic Foot and Ankle Society Hallux Metatarsophalangeal Index (AOFAS-HMI) and visual analogue scale (VAS) for pain and satisfaction].

Results There was a significant difference between patient age $(p=0.001)$, age at onset $(p<0.001)$ and AOFAS-HMI $(p=0.035)$ at follow-up. Other parameters were similar in both groups.

Conclusion Regardless of BMI, the radiological outcome was comparable. Despite a significant difference in AOFAS-HMI
\end{abstract}

Marcin Domżalski

marcin.domzalski@umed.lodz.pl

1 Orthopedic and Trauma Department, Veteran's Memorial Teaching Hospital in Lodz, Medical University of Lodz, Zeromskiego 113, 90-549 Lodz, Poland

2 Department of Psychiatry, Medical University of Lodz, Lodz, Poland

3 Department of Biostatistics and Translational Medicine, Medical University of Lodz, Lodz, Poland results, pain and satisfaction level were similar. The authors agreed that high BMI has protective role in the prevalence of HV.

Keywords SCARF $\cdot$ Hallux valgus $\cdot \mathrm{BMI} \cdot$ Overweight

\section{Introduction}

Hallux valgus (HV) is the most common forefoot deformation in adults. It is characterised by medial deviation of the first metatarsal with lateral deviation and rotation of the big toe, which leads to bunion formation and difficulty walking in footwear [1]. Its prevalence, depending on the source, is between 1:100 and 1:2 in adults. Despite its common occurrence and numerous studies, there is lack of satisfactory data on HV. One of the unexplored and significant issues is the influence of body weight on the course, management and outcome of HV treatment.

Excess weight is an increasing global problem involving health and economic issues. It is known to be a risk factor for kidney and cardiovascular diseases, cancers, diabetes and sudden death. Through excessive loads on the joints, it leads to the development of osteoarthritis [2]. Each year, orthopaedic surgeons treat a growing number of overweight patients facing specific problems. This inclination impinges on the need to study different aspects of body mass and its effect on possible treatments. It is also known, that obesity worsens wound and bone healing [3]. A number of studies acknowledged the negative influence of high body mass index (BMI) on total knee replacement, such as poorer clinical outcome and higher revision rate than in normal-weight patients [4].

London et al. compared the rates of post-operative complications in obese and nonobese patients following elbow, forearm and hand surgery [5]. According to their results, 
increasing BMI in the obese group was associated with greater complication rates compared with the group with BMI within the normal range. On the other hand, in a recent paper, Stewart et al. concluded that number of complications after forefoot surgery in obese patients was comparable with the nonobese group [6]. The only difference noted was for infections; however, the authors presumed that this was due to higher incidence of diabetes in the obese than the non-obese group rather than being overweight itself. Regardless of weight, diabetic patients were associated with significantly higher rates of surgery-site infection (SSI). This finding corresponded with other recent reports $[7,8]$.

Little is known about the role of obesity in the course, management and outcome of HV. Limited results from available literature show extensive discrepancy among authors $[9$, 10]. This might be explained by selection bias because the studies were not prospectively prepared. At the time of writing this paper, no prospective study concerning the role of obesity in the course, management and outcome of HV treatment had been published. We conducted our study to address this issue.
Our aim was to investigate the association between being overweight and clinical and radiological results of SCARF osteotomy of the first metatarsal for HV correction (Table 1).

\section{Materials and methods}

This study was carried out at the University Hospital between January 2011 and December 2015 and was approved by local ethical committee (RNN/636/13/KB). During this period, 244 patients scheduled for HV surgery were reviewed. Inclusion criteria were age $>18$ years, moderate to severe HV deformation based on the Manchester scale proposed by Garrow et al. [9]. Patients who had undergone primary HV surgery and presented recurrent deformation were excluded. Other primary exclusion criteria were underweight $(\mathrm{BMI}<18.5)$, cigarette smoking, diabetes mellitus, rheumatoid arthritis, lower limb joint instability or axis deformity, recent trauma, neurological conditions, peripheral vascular disease and severe first metatarsophalangeal-joint arthrosis. These were based on the
Table 1 Patient dataset: continuous variables presented as medians with interquartile ranges (IQR) and nominal variables presented as numbers with appropriate percentage

\begin{tabular}{|c|c|c|c|}
\hline Parameter $(n)$ & G1 $(n 71(53 \%))$ & $\mathrm{G} 2(n 62(47 \%))$ & $P$ value \\
\hline BMI $\left(\mathrm{kg} / \mathrm{m}^{2}\right)$ & $23.05(20.83-23.96)$ & $27.37(26.35-29.67)$ & \\
\hline Age (years) & $52.0(34.0-61.0)$ & $61.0(57.0-67.0)$ & $<0.001$ \\
\hline Age at onset (years) & $32.0(16.0-43.0)$ & $44.5(37.0-54.0)$ & $<0.001$ \\
\hline Symptoms-to-surgery period (years) & $15.0(10-25.0)$ & $15.0(8.0-20.0)$ & 0.496 \\
\hline Side (left/right) & $31: 40(43.7 \%)$ & $32: 30(51.6 \%)$ & \\
\hline \multicolumn{4}{|l|}{ AOFAS-HMI } \\
\hline - Presurgery & $47.0(34.0-55.0)$ & $46.0(30.0-52.0)$ & 0.252 \\
\hline - 2 years' follow-up & $80.0(70.0-90.0)$ & $75.0(67.0-85.0)$ & 0.035 \\
\hline - Difference (post-presurgery) & $33.0(26.0-40.0)$ & $31.5(22.0-41.0)$ & 0.565 \\
\hline \multicolumn{4}{|l|}{ HVA } \\
\hline - Presurgery & $39.0(27.0-36.0)$ & $33.0(28.0-36.0)$ & 0.325 \\
\hline - 2 years' follow-up & $14.0(12.0-16.0)$ & $14.0(12.0-18.0)$ & 0.247 \\
\hline - Difference (post-presurgery) & $17.0(15.0-21.0)$ & $17.0(14.0-22.0)$ & 0.786 \\
\hline \multicolumn{4}{|l|}{ IMA } \\
\hline - Presurgery & $13.0(11.0-15.0)$ & $13.0(11.0-15.0)$ & 0.652 \\
\hline - 2 years' follow-up & $8.0(7.0-9.0)$ & $9.0(7.0-10.0)$ & 0.113 \\
\hline - Difference (post-presurgery) & $5.0(3.0-6.0)$ & $4.0(3.0-6.0)$ & 0.552 \\
\hline \multicolumn{4}{|l|}{ VAS for pain } \\
\hline - Presurgery & $5.00(4.00-6.00)$ & $5.00(4.00-6.00)$ & 0.456 \\
\hline - 2 years' follow-up & $2.00(1.00-3.00)$ & $2.00(1.00-2.00)$ & 0.290 \\
\hline - Difference (post-presurgery) & $3.00(2.00-4.00)$ & $3.00(2.00-4.00)$ & 0.371 \\
\hline \multicolumn{4}{|l|}{ Complications } \\
\hline - SSI & $2(2.82 \%)$ & $2(3.23 \%)$ & 1.000 \\
\hline - Persistent pain & $3(4.23 \%)$ & $4(6.45 \%)$ & 0.705 \\
\hline - Bone healing & $2(2.82 \%)$ & $3(4.84 \%)$ & 0.664 \\
\hline - HVA postsurgery $>19^{\circ}$ & $8(11.43 \%)$ & $15(24.19 \%)$ & 0.089 \\
\hline
\end{tabular}

$B M I$ body mass index, HVA hallas vargas, AOFAS-HMI American Orthopaedic Foot and Ankle Society Hallux Metatarsophalangeal Index, IMA intermetatarsal angle, VAS visual analogue scale, SSI surgery-site infection, GI group 1, G2 group 2 
known correlation with complication rate and clinical outcome [10]. One hundred and sixty-five patients met inclusion criteria, gave written consent to participate and were included in the study. There were only women.

Each patient was examined twice: pre-operatively, and two years after surgery, by two authors $(\mathrm{MM}, \mathrm{KN})$. During the first visit, medical data was collected: age, weight, height, comorbidities, disease course, family history, running, wearing high heels, tobacco use. All patients were examined clinically, and visual analogue scale (VAS) for pain and American Orthopaedic Foot and Ankle Society Hallux Metatarsophalangeal Index (AOFAS-HMI) scores were collected [11]. AOFAS-HMI was proven to be of high reliability and viability [12]. It comprises three sections related to pain, function and alignment assessment, with the worst score being 0 and the best 100 . Worth noting is the proven correlation of AOFAS-HMI score with radiological results obtained after surgical correction of $\mathrm{HV}$ was described [13]. VAS was used to evaluate pain, where 0 is no pain and 10 is the worst pain possible. The load-bearing anteroposterior (AP) $X$-ray was taken pre-operatively and both $\mathrm{HV}$ angle (HVA) and intermetatarsal angle (IMA) were measured. Weight and height was obtained by a single qualified nurse pre-operatively using Radwag WPT 100/200 OW personal medical scale. BMI was calculated as body mass divided by the square of body height $\left(\mathrm{kg} / \mathrm{m}^{2}\right)$. Following that, patients were classified as normal weight when BMI was 18.5-25 [group 1 (G1)] or overweight with BMI > 25) [group 2 (G2)].

SCARF corrective surgery was performed in all cases. A single IV dose of antibiotic prophylaxis was administered preoperatively (second-generation cephalosporin). Anaesthesia was induced via the subarachnoid space, after which a tourniquet was applied. The surgical technique was previously described by Weil [14]. Briefly, following a medial approach, a longitudinal $Z$-shape first metatarsal shaft osteotomy splits the bone into two segments. The transverse cut was made horizontally, distal dorsal and proximal plantar. Next, the plantar segment was displaced laterally and the dorsal medially for IMA to be reduced. Additional HVA decrease was obtained by rotating the head of the first metatarsal. When satisfactory adjustment was obtained, bone segments were fixed with two 2.5-mm cannulated cortical screws (Pyxis, EOS, France). Afterwards, all patients underwent intermetatarsal-space soft-tissue release, which included lateral capsulotomy, intermetatarsal ligamentotomy and adductor halluces tenotomy. Skin closure with continuous everting mattress suture was performed and an elastic bandage applied. A Barouk shoe to off-load pressure from the forefoot was used for six weeks; no crutches were necessary, and immediate motion was begun. Patients were given enoxaparin ( $40 \mathrm{mg}$ SC daily) prophylaxis for seven days post-operatively. Tramadol plus paracetamol was prescribed for the pain control in the early post-operative stage. No nonsteroidal anti-inflammatory drugs were used. All patients were discharged home after one to two days, and the stitches were removed after 14 days. Patients who had a procedure other than SCARF osteotomy, such as Akin proximal phalanx correction or hammer toe correction, were excluded from the study $(n=59)$. Two patients were lost to follow-up.; the final number of patients who reached the two years' follow-up was 133 (see Fig. 1).

Records were collected pre-operatively, and at the two year follow-up examination, load-bearing X-ray, VAS for pain, subjective foot appearance and AOFAS-HMI data were collected. In addition, patients were again asked if they were willing to give consent again, knowing the course and results of the treatment beforehand. Load-bearing AP X-rays were again taken during the final visit; HVA and IMA were both remeasured. The angles were evaluated as previously described by Coughlin et al. [15]. All radiograms were examined by the same professional (MM). Clinical status was measured again with AOFAS-HMI [11] and VAS for pain. VAS for

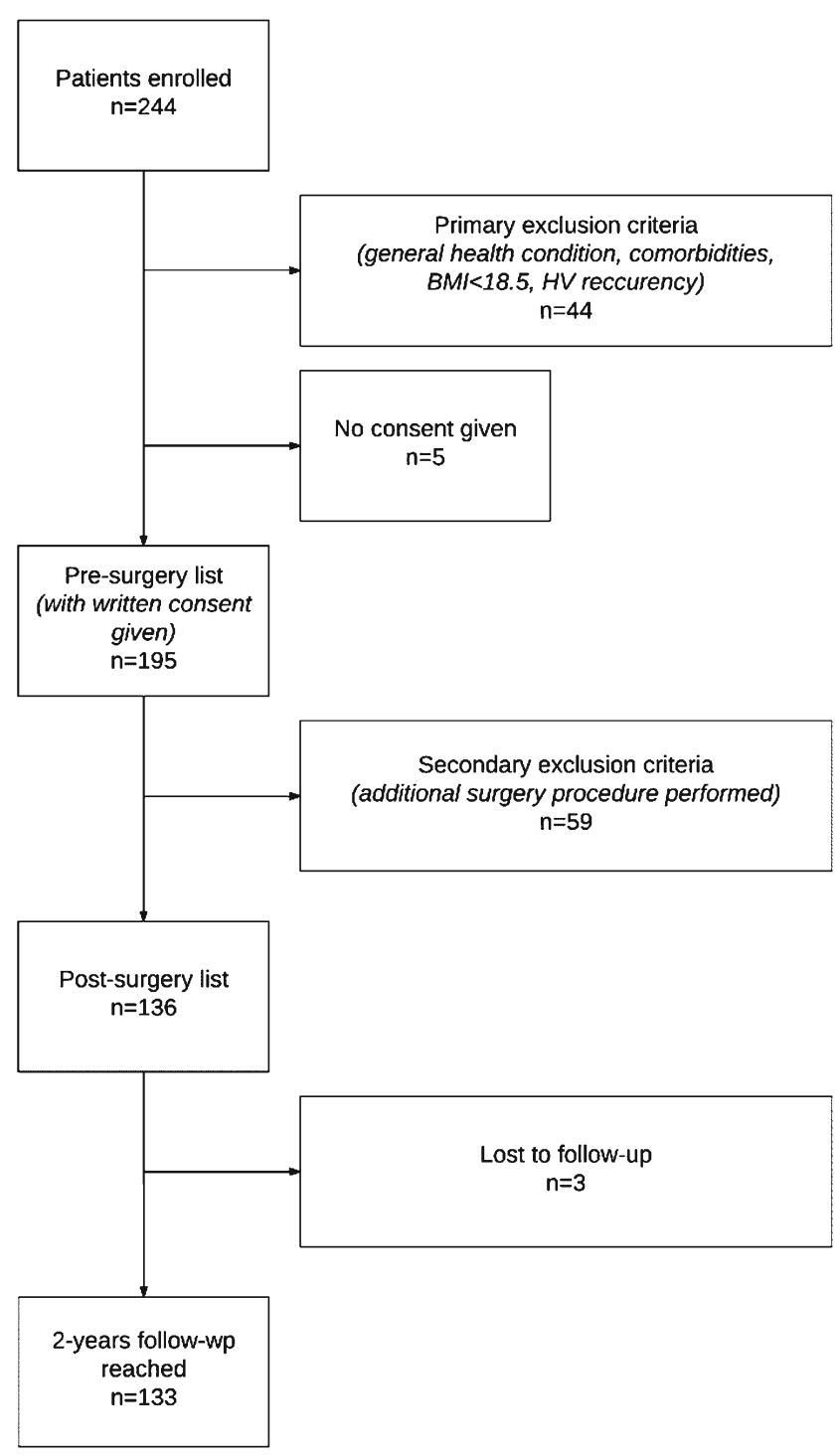

Fig. 1 Patient enrollment 
satisfaction with the treatment was collected: 0 for extremely satisfied and 10 for extremely dissatisfied. Additionally, all complications reported by patients during the two year follow-up were recorded, validated and analysed.

\section{Statistical analysis}

Nominal variables are shown as numbers with appropriate percentage whereas continuous variables are presented as medians with interquartile ranges (IQR). Chi-square tests, with appropriate corrections, were used to test associations between categorical variables. Statistica Software version 12.5 (StatSoft, Tulsa, OK, USA) was used for the analyses. $P$ values $<0.05$ were considered as statistically significant.

\section{Results}

Among 133 patients with symptomatic HV 62 (47\%) were overweight (G2) and the remaining 71 (53\%) had normal BMI (G1). The average BMI in the whole cohort was 25.00 $\pm 3.60 \mathrm{~kg} / \mathrm{m}^{2}$, median values in G1 and G2 were $23.05 \mathrm{~kg} / \mathrm{m}^{2}$ (IQR 20.83-23.94 $\mathrm{kg} / \mathrm{m}^{2}$ ) and $27.37 \mathrm{~kg} / \mathrm{m}^{2}$ (IQR 26.35$29.67 \mathrm{~kg} / \mathrm{m}^{2}$ ), respectively. BMI range is presented in Fig. 2. Median age in $\mathrm{G} 2$ was nine years older than in G1: 61.0 years, (IQR 57.0-67.0) vs. 52.0 years (IQR: 34.0-61.0), $p<0.001$ ), which corresponds with Polish population trends. The onset of symptoms occurred earlier in G1 patients: 32.0 years (IQR: $16.0-43.0$ ) vs. 44.5 years (IQR: $37.0-54.0$ ); $p<0.001$ ), (Fig. 3). The period between first symptoms and orthopaedic examination did not differ between groups $(p=0.496)$. There were slight differences in terms of affected side: in G1, the right side was affected more often (56\%), while in G2, the left foot lesions were more prevalent (52\%).

We saw no significant differences in radiological parameters between both groups. Median HVAs at both timepoints are presented in Fig. 4. On pre-operative X-rays, median HVA was 33.0 (IQR 27.0-36.0) while median IMA was 13.0 (IQR 11.0-15.0). At follow-up, values were 14.0 (IQR 12.0-17.0) and 8.50 (IQR 7.0-10.0), respectively. Pre-operative total AOFAS-HMI (Fig. 5) was comparable between groups: 47.0 (IQR 34.0-55.0) in G1 vs. 46.0 (IQR 30.0-52.0) in G2; $p=0.252$. There was a significant difference in AOFASHMI score at the follow-up, with non-obese patients achieving better results: 80.0 (IQR 70.0-90.0) in G1 vs. 75.0 (IQR 67.0 $85.0) ; p=0.035$ ). When splitting it into partial results, we found a significant correlation in activity score on primary AOFAS-HMI $(p<0.001)$, and activity and shoes score in function at the follow-up ( $p=0.024$ and $p=0.004$, respectively) in favour of G1.

Complications registered during the follow-up were as follows: SSI, persistent pain, inadequate correction and bonehealing problems (delayed union, first metatarsal fracture and metal migration). Post-operative SSI was noted in $2.82 \%(n=2)$ patients from G1 and 3.23\% $(n=2)$ in G2 $(p=1.000)$. All infections were successfully treated with antibiotics, and no chronic inflammation or fistula formation was reported. No patient required surgical debridement. Persistent pain was noted in $4.23 \%(n=3)$ and $6.45 \%(n=4)$ patients, respectively. Bone-healing problems were also similar $(2.82 \%$ and $4.84 \%, p=0.664)$. There was one fracture in each group that occurred due to noncompliance. We also reported one metal migration in $\mathrm{G} 2$ and one delayed union in both groups. The highest complication rate was represented by inadequate
Fig. 2 Body mass index (BMIC) spread: horizontal bars represent median, whiskers represent interquartile range and dots represent raw data

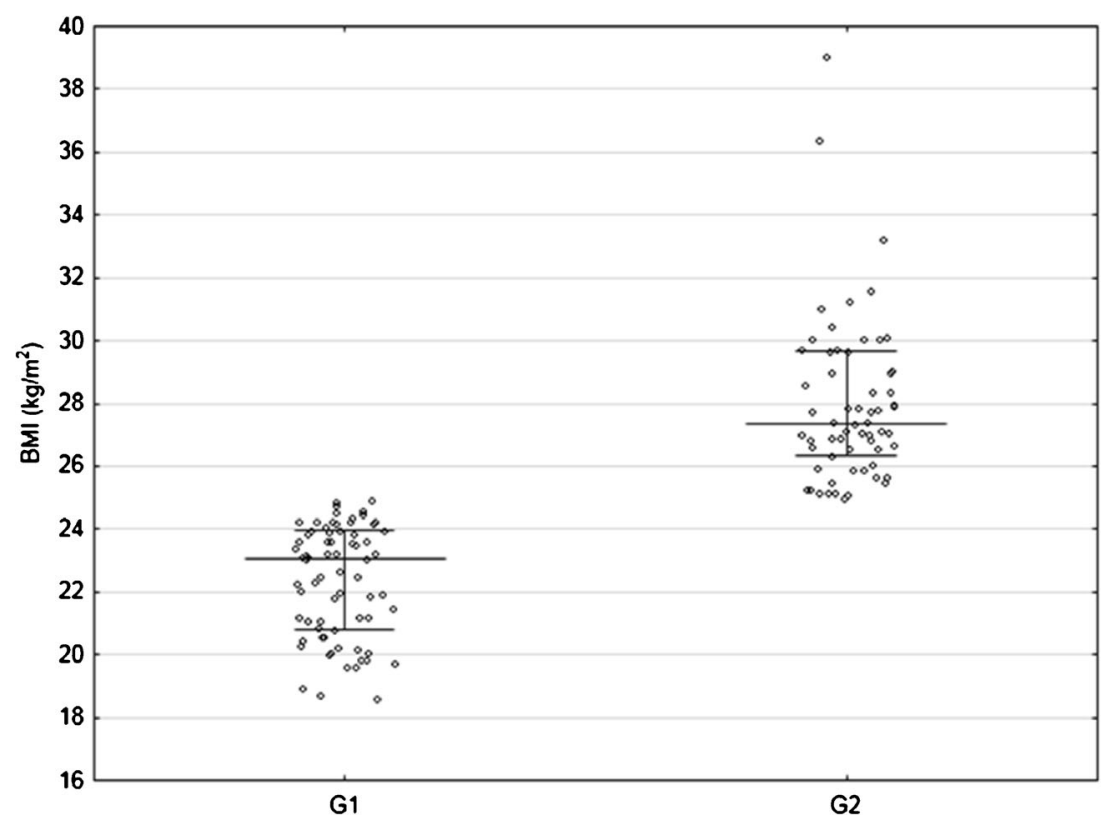


Fig. 3 Symptom onset: horizontal bars represent median, whiskers represent interquartile range and dots represent raw data

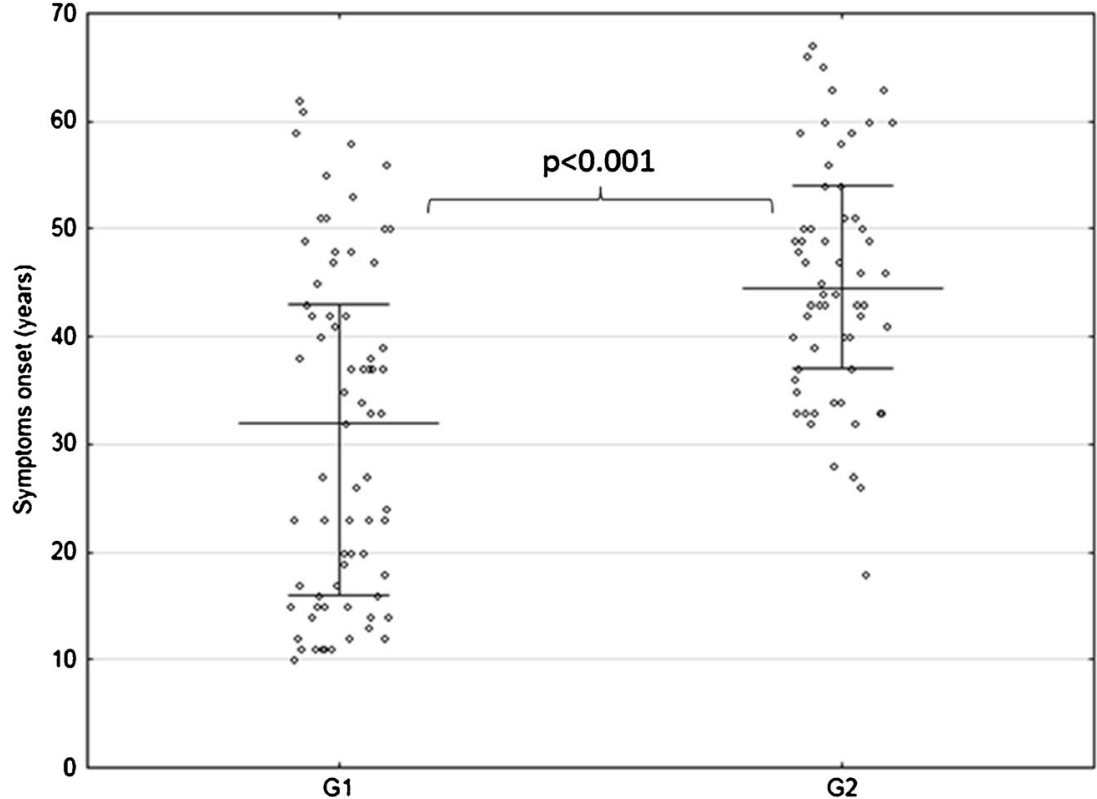

correction (HVA > 19), which was observed in 15 patients overall $(11.28 \%)$. Although we noticed $9.85 \%(n=7)$ in $\mathrm{G} 1$ and $12.90 \%(n=8)$ in G2, no significant difference was found $(p=0.089)$. Results of VAS for pain were similar between groups at both timepoints. CMedian pain reduction level was 3.0 units in both groups $(p=0.371)$.

Patients' subjective foot appearance between groups in preoperative evaluation was identical $(3.0, p=1.000)$, and at two years' follow-up, no significant differences were found (1.4 vs. $1.5, p=0.884)$. Median VAS for satisfaction score was 3.0 (IQR 2.00-4.00) in the normal weight group and 3.0 (IQR $2.00-3.00)$ in the overweight group $(p=0.339)$. Patients in both groups gave their secondary consent for treatment (92\% vs. $89 \%, p=0.797)$.

\section{Discussion}

To the authors' knowledge, this article is the first report regarding the role of being overweight in the HV SCARF surgery based on a prospective study. The mainstay of HV treatment is reduction or complete abolition of pain and difficulty wearing shoes, which are the most acute symptoms. The secondary goal is to improve foot functionality and aesthetics.
Fig. 4 Hallus vargus (HVA) results: horizontal bars represent median, whiskers represent interquartile range. Raw data from the first timepoint (before surgery) is presented as dots, while raw data from the second timepoint (2-years' follow-up) is presented as triangles

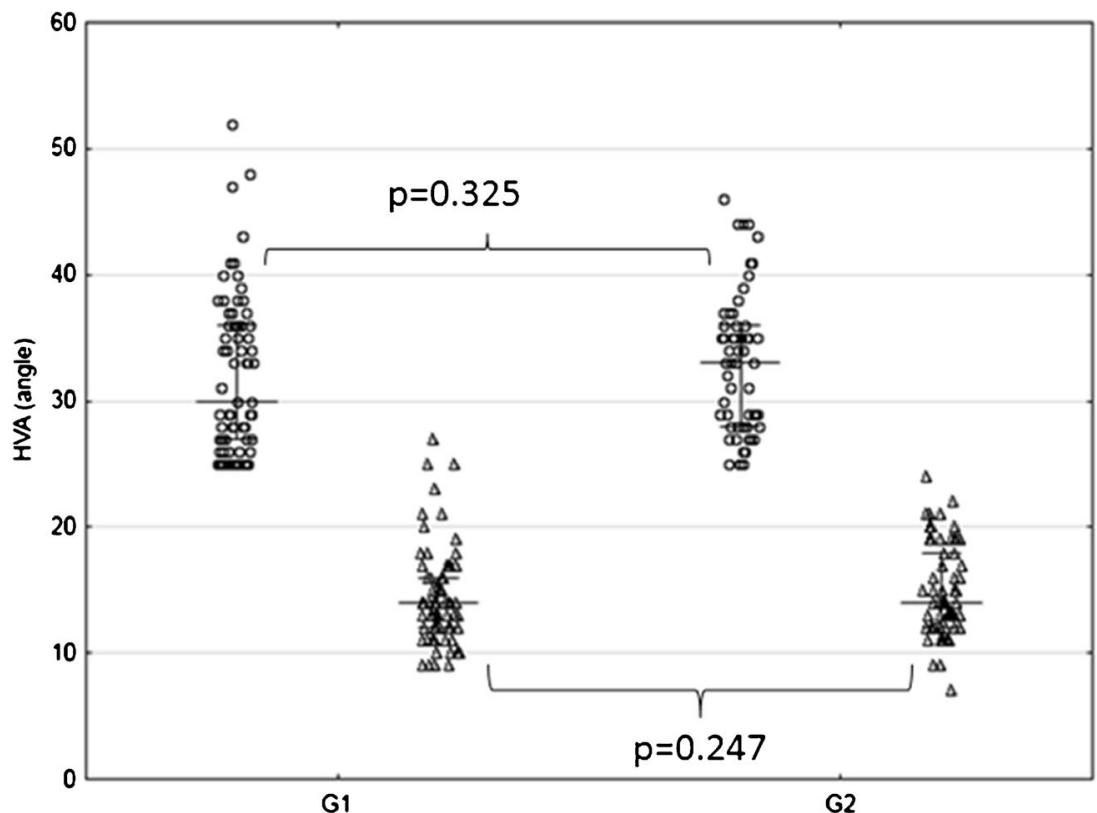


Fig. 5 American Orthopaedic Foot and Ankle Society Hallux Metatarsophalangeal Index (AOFAS-HMI) results: horizontal bars represent median, whiskers represent interquartile range. Raw data from the first timepoint (before surgery) is presented as dots, while raw data from the second timepoint (2years' follow-up) is presented as triangles

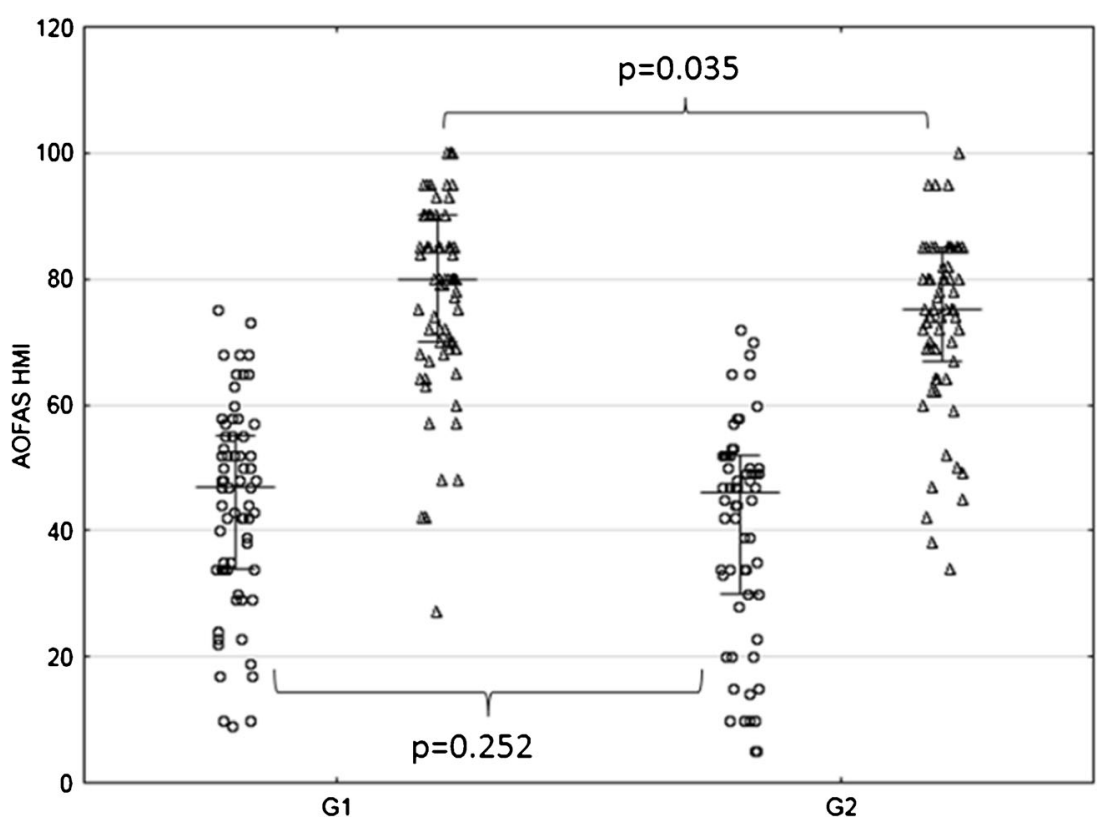

This study aimed at comparing differences of prevalence and outcome in consideration of BMI.

The overweight group was represented by patients nine years older on average than those in the normal-weight group. According to the Polish Central Statistical Office, this observation meets national trends of increasing weight with age. More interesting is the difference in age at symptom onset between the groups. We noted a strong prevalence of $\mathrm{HV}$ symptoms presenting at a younger age in patients within normal BMI range. Conversely, median onset in overweight patients was 12.5 years later. The influence of BMI on HV incidence in the literature is inconsistent. In 1990, Wallace et al. reported that high BMI has a protective role in the development of HV [16]. A retrospective study by Frey and Zamora investigated the association of the incidence of foot and ankle complaints with BMI in 1411 patients [17]. They observed that although being overweight brings a greater chance of tendinitis, plantar fasciitis and osteoarthritis, normal-weight patients are more likely to develop HV. Conversely, Cho et al. reported that patients with higher BMI are more prone to develop HV [13]. In 2010, the MOBILIZE Boston Study reported that the prevalence of $\mathrm{HV}$ in women decreases with increased BMI [18]. Our results were similar, with higher BMI corresponding with older age at symptoms onset, and confirm the protective role of being overweight on the development of HV in women. Dufour and Golightly stated that great-toe deformation was inversely associated with higher BMI in both sexes $[19,20]$. On the other hand, a recent study by Roddy et al. presented no association between HV and BMI [17]. A weak point of these studies was lack of radiological assessment. An evaluation using a visual scale (or a diagram used in this study) has legitimised application in clinical practice but is likely to be less precise in research dependent on precise data. We believe that higher body mass only postpones the symptom onset and suggest further cohort studies with regard to HV prevalence in BMI groups of different age and sex.

The results of VAS for satisfaction showed that most patients were satisfied with the surgical outcome and therapeutic course, with similar median level of 3.0. Finally, patients asked to give consent again for therapy responded positively in $\sim 90 \%$ of cases. As there was no significant difference between groups, we believe that the described method of managing $\mathrm{HV}$ is satisfactory for all patients regardless of BMI.

We observed similar radiological results between groups at both timepoints. We suggest that the close to significant gain in the number of HVA $>19$ at the follow-up $(p=0.089)$ in overweight patients can be explained by worse intra-operative access and tougher, more difficult, rearrangement of cut bone segments than in the normal-weight group due to excessive fat tissue. Similar observations were mentioned in the literature regarding different orthopaedic surgeries. Chen et al. reported a sevenfold increase in repeat surgery due to obesity in their two year postoperative studies [21]. It is questionable, however, whether the phenomenon occurred regardless of the enrolment of diabetic patients in their study or because of it. Future largescale studies are required to determine the reason.

It is debatable whether the distal metatarsal articulation angle (DMAA) has any influence on complication rate. We did not obtain DMAA data, as we met the $\pm 3^{\circ}$ diversity range between different researchers. This observation is similar to that in number of independent studies [22-24].

While our mean pre-operative AOFAS-HMI results were comparable between groups, scores were slightly lower in the obese group at follow-up. The explanation lies in partial results, where we observed a significant correlation between activity and shoes score in the Function section in favour of 
G1. We believe this is due to the psychological aspect of patients with higher BMI, leading to avoidance of physical activity and the pursuit of comfort, which is described widely in the literature [25]. Excessive weight is a cause of musculoskeletal pain; previous studies prove the association of obesity with low-back, knee and foot pain. In 2012, Butterworth et al. presented a systematic review investigating the impact of elevated BMI on foot disorders [26], particularly a significant association between obesity and chronic foot pain - either plantar or nonspecific. In our studies, VAS for pain associated with HV deformity was similar between groups at both timepoints $(p=0.456$ and $p=0.290)$., with median improvement being 3.0. Nevertheless, AOFAS-HMI pain-related partial results show a significant correlation between lower scores in both groups at the follow-up. Interestingly, mean VAS pain scores were 0.2 lower than in the normal-weight group. We believe the reason lies in patient perception on both scales, which in VAS focuses on pain level (toughness) and in AOFAS on pain intensity in time.

There was similar incidence of SSI and other complications. The only issue close to significance was inadequate correction in the overweight group, as discussed above. Stewart et al. suggested that significant correlation of SSI incidence and diabetes mellitus can be obtained with a higher-power study [6]. Conversely, Chen et al. observed no difference in complication rate when assessing the presence of diabetes mellitus (DM) or BMI [21]. Wukich suggested that the higher SSI rate in diabetic patients decreases if the disease is well managed [7, 8]. Our study did not include diabetic patients in order to avoid its hypothetical influence on outcomes. We observed four cases of SSI and four ( two per group, $p=1.000$ ) of delayed bone healing, all of which were successfully treated by the end of this study. Although seven patients complained of persistent forefoot pain at the final follow-up, no statistical correlation was found between groups, with no significant effect on VAS pain score. As the single cases of metal migration and first metatarsal fracture were due to patient noncompliance, we were unable to study possible relations. Interestingly, long-term clinical and radiological results and complications observed in both groups were similar to those presented by other authors studying long-term issue [27-29]. Therefore, we see no influence of BMI on SSI, delayed bone healing or persistent pain rates after HV surgery.

Bock et al. [29] and Jeuken et al. [28] presented results of SCARF osteotomy in their two year and 14-year follow-up studies, respectively. They observed a gradual drop in clinical and radiological outcome in both groups with time post-surgery. As their two year follow-up results were similar to ours, we expect similar decline. Interestingly, in the SCARF group, $78 \%$ recurrence was reported by Jeuken after 14 years. Compared with our studies, this number is very high and we do not expect to come close over a similar period. The reason seems to be different characteristics of recurrence (HV angle $>15^{\circ}$ vs. $\mathrm{HVA}>19^{\circ}$ ).

Weil and Bowen, in their epic paper on SCARF for HV surgery, pointed out that disparity in long-term results may occur due to a steep surgical learning curve [27]. They noted that some authors observed as much as $35 \%$ complication rate in their primary studies and as few as $1 \%$ in studies performed a few years later. Whereas adding supplementary data from different timepoints of the learning curve could result in a huge divergence of findings, it seems appropriate to analyse the same group of patients for mid- and long-term results.

In conclusion, this study confirms that SCARF osteotomy can be recommended for treating moderate to severe $\mathrm{HV}$ regardless of body weight. It results in a satisfactory correction of angular deformities, good clinical outcome, reduced pain and high patient satisfaction. There was no significant difference in complication rate between obese patients and those of normal BMI.

\section{Compliance with ethical standards}

Conflict of interest The authors declare no conflict of interest with this project.

Funding There was no funding source for this study.

Ethical approval This study was approved by Local Ethical Committee (RNN/636/13/KB).

Informed consent Informed consent was obtained from all individual participants included in the study.

Open Access This article is distributed under the terms of the Creative Commons Attribution 4.0 International License (http:// creativecommons.org/licenses/by/4.0/), which permits unrestricted use, distribution, and reproduction in any medium, provided you give appropriate credit to the original author(s) and the source, provide a link to the Creative Commons license, and indicate if changes were made.

\section{References}

1. Nery C, Coughlin MJ, Baumfeld D, Ballerini FJ, Kobata S (2013) Hallux valgus in males-part 1: demographics, etiology, and comparative radiology. Foot Ankle Int 34(5):629-635

2. Sharma L, Lou C, Cahue S, Dunlop DD (2000) The mechanism of the effect of obesity in knee osteoarthritis: the mediating role of malalignment. Arthritis Rheum 3(1):568-575

3. Stevens SM, O'Connell BP, Meyer TA (2015) Obesity related complications in surgery. Curr Opin Otolaryngol Head Neck Surg 23(5):341-347

4. Collins RA, Walmsley PJ, Amin AK, Brenkel IJ, Clayton RA (2012) Does obesity influence clinical outcome at nine years following total knee replacement? J Bone Joint Surg (Br) 94(10): 1351-1355

5. London DA, Stepan JG, Lalchandani GR, Okoroafor UC, Wildes TS, Calfee RP (2014) The impact of obesity on complications of elbow, forearm, and hand surgeries. J Hand Surg [Am] 39(8):15781584 
6. Stewart MS, Bettin CC, Ramsey MT, Ishikawa SN, Murphy GA, Richardson DR, Tolley EA (2016) Effect of obesity on outcomes of forefoot surgery. Foot Ankle Int 37(5):483-487

7. Wukich DK, McMillen RL, Lowery NJ, Frykberg RG (2011) Surgical site infections after foot and ankle surgery: a comparison of patients with and without diabetes. Diabetes Care 34(10):22112213

8. Wukich DK, Crim BE, Frykberg RG, Rosario BL (2014) Neuropathy and poorly controlled diabetes increase the rate of surgical site infection after foot and ankle surgery. J Bone Joint Surg Am 96(10):832-839

9. Garrow AP, Papageorgiou A, Silman AJ, Thomas E, Jayson MI, Macfarlane GJ (2001) The grading of hallux valgus. The Manchester scale. J Am Podiatr Med Assoc 91(2):74-78

10. den Broeder AA, Creemers MC, Fransen J, de Jong E, de Rooij DJ, Wymenga A, de Waal-Malefijt M, van den Hoogen FH (2007) Risk factors for surgical site infections and other complications in elective surgery in patients with rheumatoid arthritis with special attention for anti-tumor necrosis factor: a large retrospective study. J Rheumatol 34(4):689-695

11. Kitaoka HB, Alexander IJ, Adelaar RS, Nunley JA, Myerson MS, Sanders M (1994) Clinical rating systems for the ankle-hindfoot, midfoot, hallux, and lesser toes. Foot Ankle Int 15(7):349-353

12. Ibrahim T, Beiri A, Azzabi M, Best AJ, Taylor GJ, Menon DK (2007) Reliability and validity of the subjective component of the American orthopaedic foot and ankle society clinical rating scales. J Foot Ankle Surg 46(2):65-74

13. Thordarson D, Ebramzadeh E, Moorthy M, Lee J, Rudicel S (2005) Correlation of hallux valgus surgical outcome with AOFAS forefoot score and radiological parameters. Foot Ankle Int 26(2):122127

14. Weil LS (2000) SCARF osteotomy for correction of hallux valgus. Historical perspective, surgical technique, and results. Foot Ankle Clin 5(3):559-580

15. Coughlin MJ, Jones CP (2007) Hallux valgus: demographics, etiology, and radiographic assessment. Foot Ankle Int 28(7):759-777

16. Wallace WA, Kilmartin TE (1990) Predicting hallux abducto valgus. J Am Podiatr Med Assoc 80(9):509-510

17. Roddy E, Muller S, Rome K, Chandratre P, Hider SL, Richardson J, Blagojevic-Bucknall M, Mallen CD (2015) Foot problems in people with gout in primary care: baseline findings from a prospective cohort study. J Foot Ankle Res 8:31
18. Nguyen US, Hillstrom HJ, Li W, Dufour AB, Kiel DP, Procter-Gray E, Gagnon MM, Hannan MT (2010) Factors associated with hallux valgus in a population-based study of older women and men: the MOBILIZE Boston Study. Osteoarthr Cartil 18(1):41-46

19. Golightly YM, Hannan MT, Dufour AB, Renner JB, Jordan JM (2015) Factors associated with hallux valgus in a communitybased cross-sectional study of adults with and without osteoarthritis. Arthritis Care Res 67(6):791-798

20. Dufour AB, Casey VA, Golightly YM, Hannan MT (2014) Characteristics associated with hallux valgus in a populationbased foot study of older adults. Arthritis Care Res 66(12):18801886

21. Chen JY, Lee MJ, Rikhraj K, Parmar S, Chong HC, Yew AK, Koo KO, Singh Rikhraj I (2015) Effect of obesity on outcome of hallux valgus surgery. Foot Ankle Int 36(9):1078-1083

22. Piqué-Vidal C, Vila J (2009) A geometric analysis of hallux valgus: correlation with clinical assessment of severity. J Foot Ankle Res 2: 15

23. Cakmak G, Kanatlı U, Kılınç B, Yetkin H (2013) The effect of pronation and inclination on the measurement of the hallucal distal metatarsal articular set angle. Acta Orthop Traumatol Turc 47(5): 354-358

24. Jastifer JR, Coughlin MJ, Schutt S, Hirose C, Kennedy M, Grebing B, Smith B, Cooper T, Golano P, Viladot R, Doty JF (2014) Comparison of radiographic and anatomic distal metatarsal articular angle in cadaver feet. Foot Ankle Int 35(4):389-393

25. Al-Hazzaa HM, Abahussain NA, Al-Sobayel HI, Qahwaji DM, Musaiger AO (2012) Lifestyle factors associated with overweight and obesity among Saudi adolescents. BMC Public Health 12:354

26. Butterworth PA, Landorf KB, Smith SE, Menz HB (2012) The association between body mass index and musculoskeletal foot disorders: a systematic review. Obes Rev 13(7):630-642

27. Weil L Jr, Bowen M (2014) SCARF osteotomy for correction of hallux abducto valgus deformity. Clin Podiatr Med Surg 31(2):233246

28. Jeuken RM, Schotanus MG, Kort NP, Deenik A, Jong B, Hendrickx RP (2016) Long-term follow-up of a randomized controlled trial comparing SCARF to chevron osteotomy in hallux valgus correction. Foot Ankle Int 37(7):687-695

29. Bock P, Kluger R, Kristen KH, Mittlböck M, Schuh R, Trnka HJ (2015) The SCARF osteotomy with minimally invasive lateral release for treatment of hallux valgus deformity: intermediate and long-term results. J Bone Joint Surg Am 97(15):1238-1245 